\title{
Sonographic incidence of tendon microtears in athletes with chronic Achilles tendinosis
}

\author{
W W Gibbon, J R Cooper, G S Radcliffe
}

\begin{abstract}
Objective-To assess the number and distribution of tendon microtears in asymptomatic controls and athletes with chronic Achilles tendinitis or partial thickness tears using high resolution ultrasound.

Methods-The mean number of microtears in three random tendon cross sections were recorded per tendon third in 19 asymptomatic volunteers, 16 athletes with symptomatic chronic Achilles tendinitis, and eight athletes with partial Achilles tendon rupture.

Results-Microtears were most numerous in the middle third section of the Achilles tendon. Some $67 \%$ of tendons in the control group had no microtears, and $28 \%$ showed a single microtear. Only $18 \%$ of the athletes with chronic Achilles tendinitis and none of the athletes with partial tendon rupture were without microtears in the middle third of their Achilles tendon. Of the tendons with chronic tendinitis, $13 \%$ had more than three microtears per section which increased to $87 \%$ in tendons exhibiting partial rupture. Conclusions-There appears to be an association between microtear formation and Achilles tendon rupture.

(Br F Sports Med 1999;33:129-130)
\end{abstract}

Keywords: tendon; Achilles tendon; Achilles tendinitis; Achilles tendinosis; microtears; rupture

Ultrasound is commonly used to investigate Achilles tendon disease. ${ }^{1}$ The tendon's superficial position and easy accessibility allows very detailed images to be obtained using high frequency transducers. Occasionally small foci of low echogenicity are found within normal tendons. ${ }^{2}$ These have been thought to represent microtears or small areas of altered/

Department of Radiology, The General Infirmary at Leeds, United Kingdom W W Gibbon J R Cooper

Department of Orthopaedics G S Radcliffe

Correspondence to: Dr J R Cooper, Specialist Registrar, Department of Radiology, General Infirmary at Leeds, Great George Street, Leeds LS1 3EX, United Kingdom.

Accepted for publication 26 November 1998 degenerate tendon collagen. More conglomer-

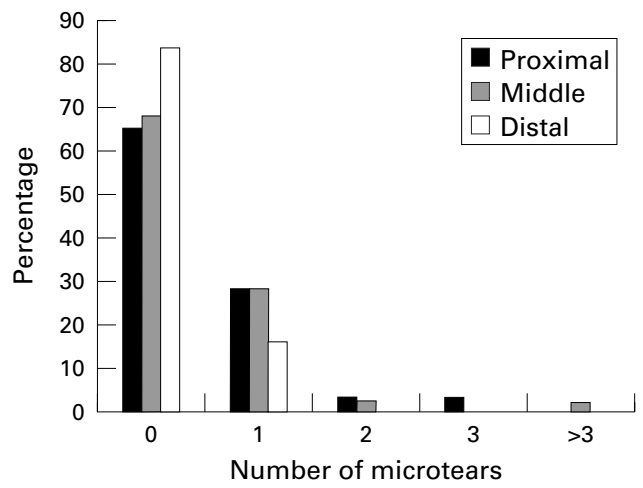

Figure 1 Percentage of microtears per tendon third in normal asymptomatic volunteers $(n=38$ heels). ate low echogenicity foci are present in patients with chronic Achilles tendinosis. It is postulated that these microdefects accumulate to produce the larger conglomerate areas of tendon degeneration.

The aim of the current study series was to assess the distribution in asymptomatic volunteers and symptomatic athletes of these Achilles tendon microdefects as disclosed by high resolution real time ultrasound.

\section{Methods}

A quantitative assessment was made of the number and distribution of Achilles tendon microtears in 19 asymptomatic volunteers (38 tendons), 16 athletes with symptomatic chronic Achilles tendinitis (16 tendons), and 8 athletes with partial Achilles tendon rupture (eight tendons). The control group were chosen to match the sex and age distribution of the symptomatic groups. The symptomatic atheletes were all refered for ultrasound assessment of their Achilles tendon with a clinical diagnosis of possible partial tendon rupture, and then subdivided into tendinitis and partial tendon rupture groups using sonographic criteria.

All patients were examined by the same experienced musculoskeletal sonologist using a 10-5 $\mathrm{MHz}$ linear array transducer (HDI 3000; Advanced Technology Laboratories, Bothel, WA, USA). The heels were examined with patients in a prone position and their heels overhanging the examination couch so that the ankles were in full dorsiflexion in order to avoid bow-stringing of the Achilles tendon. No stand off medium was required. The heels were examined in transverse and longitudinal plane and the distribution of microtears recorded. For assessment of the incidence and distribution of tendon microtears, the Achilles tendons were nominally divided into thirds according to overall tendon length. Three transverse images were obtained for each tendon third and the mean value taken as representative of that section.

\section{Results}

Achilles tendon microdefects or microtears were occasionally seen sonographically in the Achilles tendons of normal asymptomatic volunteers (fig 1). When such microdefects occur, only single foci of abnormality are usually present and these tend to be more common in the proximal two thirds of the tendon. In chronic Achilles tendinitis, the percentage of athletes with one or more microdefects significantly increases in the proximal two thirds of the Achilles tendon although the increase in the distal third is less marked (fig 2). All athletes with partial Achilles tendon rupture have three or more microtears per section in the middle 


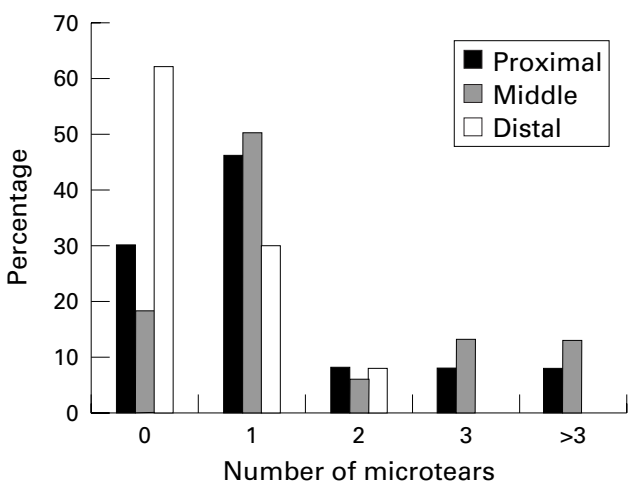

Figure 2 Percentage of microtears per tendon third in patients with chronic Achilles tendinitis ( $n=16$ heels).

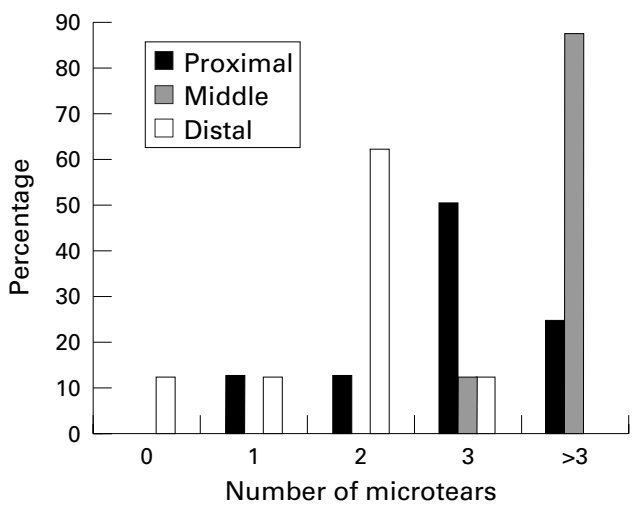

Figure 3 Percentage of microtears per tendon third in patients with partial rupture of the Achilles tendon ( $n=8$ heels).

third of the tendon away from the actual site of rupture (fig 3). In these athletes there is also a smaller increase in microtears in the distal and proximal thirds of the Achilles tendon.

\section{Discussion}

The repetitive trauma sustained in athletic pursuits causes degenerative changes in the Achilles tendon, presumably as the result of mechanical microtrauma. ${ }^{3}$ These appear to increase in number in diseased tendons. One explanation of these results is that such microtears in low numbers occur as a function of cyclical injury and repair in normal tendons. With exercise the number of such microdefects increases. Under normal circumstances these would be expected to be rapidly repaired. In these circumstances, the local inflammation reflects a reparative response rather than disease. Normal asymptomatic tendons in non-athletic individuals will exhibit occasional small hypoechoic foci on sonographic examination which may reflect either microdefects or foci of altered collagen architecture.

These results suggest that the tendon is not a static tissue but is constantly remodelling itself by a process of minor injury and repair in an analogous way to bone remodelling in accordance with the forces applied (Wolfe's Law). ${ }^{4}$ If there is increased cyclical stressing of the tendon, then it would seem logical that the number of such "microtears" increase, resulting in tendon hypertrophy. This would explain why an athlete's tendon is often thicker in cross section than a comparable non-athlete's tendon. Such a response would therefore represent a natural adaptation to the applied deforming/ tensile forces similar to the increased bone mineral density seen in the femoral necks of athletes compared with the general population. The number of sonographically demonstrable microtears in this study were found to increase in tendons exhibiting other sonographic features of tendon degeneration consistent with chronic Achilles tendinosis. These microtears appear to accumulate particularly in the middle third of the tendon - that is, in the region of a relative vascular watershed. ${ }^{5}$ It is possible that this accumulation reflects a reduced healing capacity in this region as the result of a relatively low local tissue $\mathrm{PO}_{2}$. The blood supply in the middle third of the tendon is primarily via the paratenon vessels. Injury to this vascular supply as the result of repetitive paratendinitis or failure of vessels to adequately penetrate the centre of a hypertrophied tendon may result in poor tendon healing in athletes. Therefore, in athletes, Achilles tendon degeneration may be accelerated as the result of both increased microtear production and decreased rate of microdefect repair. It is perhaps not surprising that the number of these microtears is considerably increased adjacent to the site of partial tendon rupture. The accumulation of these microtears at one site may be expected to weaken a tendon's tensile strength sufficiently for tendon failure - that is, partial or full thickness rupture-to eventually occur. Accordingly mild tendinosis and complete tendon rupture would seem to be at the opposite ends of the same spectrum of tendon injury, with the central points on this spectrum being severe tendinosis/intrasubstance tear. If this is true then it is important that these changes are diagnosed at an early stage, raising the question of whether the use of sonographic screening should be considered in "at risk" groups such as professional athletes.

1 Maffulli N, Regine R, Angelillo M, et al. Ultrasound diagnosis of achilles tendon pathology in runners. Br F Sports Med 1987;21:158-62.

2 Martinoli C, Derchi LE, Pastorino C, et al. Analysis of echotexture of tendons with US. Radiology 1993;186:839-43.

3 Clement DB, Taunton JE, Smart GW. Achilles tendinitis and peritendinitis: etiology and treatment. Am $\mathcal{F}$ Sports Med 1984;12:179-84

4 Ogden JA. Chondro-osseous development and growth. In: Urist MR, ed. Fundamental and clinical bone physiology. Philadelphia: Lippincott, 1980:108-71.

5 Kvist M. Achilles tendon injuries in athletes. Sports Med 1994;18:173-201.

\section{Take home message}

Sonographically demonstrable microtears occur more frequently in athletes with tendonitis or partial tears than in asymptomatic controls. Microtears are probably the result of repeated trauma, and their accumulation may lead to tendon failure. There may be a role for ultrasound in identifying athletes at high risk of tendon rupture. 